\title{
Perfil dos nascidos vivos no Município de Belo Horizonte, 1992-1994
}

\section{Profile of live births in Belo Horizonte, 1992-1994}

Celeste de Souza Rodrigues 1

Helvécio Miranda Magalhães Júnior 1

Patrícia Alves Evangelista 1

Roberto Marini Ladeira 1

Sandro Laudares 2

\footnotetext{
1 Núcleo de Epidemiologia, Secretaria Municipal de Saúde de Belo Horizonte Avenida Afonso Pena 2.336, 9 o andar, Belo Horizonte, MG 30130-006, Brasil.

2 Central de Suporte da Prodabel, Secretaria Municipal de Saúde de Belo Horizonte. Avenida Afonso Pena 2.336, 9o andar, Belo Horizonte, MG, 30130-006, Brasil.
}

Abstract The Information System on Live Births - SINASC - was developed by the Health Ministry and designed to improve quality of information on live births in Brazil. It has been effectively used in Belo Horizonte since 1992. The distribution of live births was analysed in order to characterise them and to allow use of existing data to support planning, organisation and evaluation of the health activities devel oped. It was conducted by using Declarations of Live Births of newborns of mothers resident in Bel o Horizonte, in the period 1992-1994, in terms of characteristics related to labour, pregnancy and mother. We concluded that it is necessary to carry out further analyses on infant and maternal morbi dity and mortality and the costs of hospital admission for caesarean section; to give special assistance to teenage mothers; and to offer good quality antenatal care to all pregnant women. SINASC is a powerful information tool and its use should be improved and disseminated, specially its use in specific and differentiated interventions for certain population groups and small risk areas.

Key words Information Systems; Live Births; Infant-Maternal Health Care; Sinasc

Resumo O Sistema de Informações sobre Nasci dos Vivos - Si nasc - foi desenvolvi do pelo Ministério da Saúde, objetivando a mel horia da qualidade das informações sobre estas crianças, no Brasil, e vem sendo usado no Muni cípi o de Belo Horizonte, efetivamente, desde 1992. Com o objetivo de caracterizar as crianças nasci das vivas e permi tir a utilização dos dados para subsidiar o planej amento, organização e avaliação de ações de saúde desenvolvidas, foi analisada a distribui ção destas, através de todas as Declarações de Nasci dos Vivos - DN, de mães resi dentes em Bel o Horizonte, 1992/94, segundo característi cas relativas ao parto,à gravi dez e à mãe. Concluiuse haver necessi dade de estudos que aprofundem a análi se da morbi-mortalidade materna e infantil e os custos das internações por partos operatórios, por uma atenção especial às adolescentes, além de acompanhamento pré-natal de boa quali dade para todas as gestantes. O Sinasc é um instrumento poderoso de informações rotineiras, devendo ser aprimorado eo seu uso difundido, incluindo a possibilidade não só de cruzamento de dados com os de outros sistemas, espe cialmente o SIM (Sistema de Informação sobre Mortalidade) e o banco de dados de AIH (Autorização para Internação Hospitalar), como também de sua utilização para intervenções específicas e diferenciadas em determi nados grupos populacionais e mi croáreas de risco. Palavras-chave Sistemas de Informação; Nasci dos Vivos; Saúde Materno-Infantil; Sinasc 


\section{Introdução}

O Sistema de Informação Sobre Nascidos Vivos - Sinasc - é um sistema desenvolvido pelo Ministério da Saúde (MS) com o objetivo de meIhorar a qualidade das informações sobre nascidos vivos no Brasil e vem sendo implantado em várias regiões do País de maneira heterogênea.

O processo de implantação em Belo Horizonte iniciou-se no segundo semestre de 1991, quando reuniram-se técnicos do Ministério da Saúde (MS), da Secretaria de Estado da Saúde de M G (SES), da Secretaria de Estado do Planejamento de MG (SEPLAN), da Secretaria Municipal de Saúde de Belo Horizonte (SM SA), da Universidade Federal de Minas Gerais (UFMG) e dos Cartórios de Registro Civil do Município para apresentação do sistema e do respectivo software. Foi formada uma comissão com representantes das instituições relacionadas acima para coordenar as ações de implantação, que ocorreu de maneira descentralizada com responsabilização dos Distritos Sanitários pelas maternidades contidas em sua área de abrangência.

Optou-se, inicialmente, pela realização de um teste piloto, tendo sido escolhidas dez maternidades (entre públicas e privadas) de maior relevância para o Município, sendo estas distribuídas pelos nove Distritos Sanitários (DS) da cidade. O teste foi realizado no período de 15/12/ 91 a 14/02/92, tendo sido feita uma apresentação prévia do sistema aos diretores das maternidades, bem como, paralelamente, um treinamento dos técnicos que realizariam o preenchimento das Declarações de Nascimento (DN).

A partir de 15 de fevereiro de 1992, o sistema foi implantado definitivamente (após treinamento) em todas as maternidades de Belo Horizonte, totalizando trinta e uma, não tendo sido encontrados problemas relacionados a qualquer das instituições acima que inviabilizassem a continuidade do processo. O processamento, consolidação e análise dos dados também se dá de maneira descentralizada nos Distritos Sanitários, sendo que o nível central consolida e analisa para o Município, uma vez ao ano. Na metade do ano de 1994, iniciou-se o recebimento pela SMSA das Declarações de Nascidos Vivos nos Municípios de Contagem e Betim, residentes em Belo Horizonte.

\section{Objetivo}

Conhecer o número de crianças nascidas vivas e residentes no município de Belo Horizonte, traçando o seu perfil e possibilitando subsidiar o planejamento, organização e avaliação das ações de saúde desenvolvidas.

\section{Material e método}

Foram utilizadas as informações contidas no banco de dados do Sinasc dos anos de 1992, 1993 e 1994, com as informações constantes das DNs.

As terceiras vias das DNs são recolhidas semanalmente pelos técnicos dos nove Distritos Sanitários do Município, trocadas entre estes de acordo com o endereço de residência das mães e digitadas. As DNs incompletas são devolvidas às maternidades para o devido preenchimento. Em caso de partos domiciliares, em que a emissão da DN ocorre nos Cartórios, as terceiras vias são enviadas ao Núcleo de Epidemiologia da SMSA, que as repassa para digitação aos respectivos Distritos Sanitários.

Na metade do ano de 1994, o Núcleo de Epidemiologia passou a receber as DNs de nascidos vivos em outros dois municípios da região metropolitana de Belo Horizonte (Betim e Contagem) e que são residentes em BH. Estas entram no mesmo fluxo descrito anteriormente. As DNs dos nascidos em $\mathrm{BH}$ e residentes em outros municípios ficam disponíveis para estes através da Secretaria de Estado da Saúde-SES.

\section{Resultados}

É apresentado a seguir o perfil dos nascidos vivos residentes em Belo Horizonte nos anos de 1992, 1993 e 1994.

A Figura 1 apresenta a distribuição dos nascidos vivos residentes em $\mathrm{BH}$ por ano e mês. $\mathrm{O}$ menor número de nascidos vivos no ano de 1992 se deve ao fato de que a implantação em todas as maternidades só ocorreu a partir de 15/ 02/ 92, não entrando, portanto, na análise os nascidos vivos de 01/01/ 92 a 14/ 02/ 92 de 21 maternidades.

A Tabela 1 mostra a distribuição destes por ano e por Distrito Sanitário de residência das mães. A diferença que se observa em relação ao total de nascidos vivos nos três anos pode ser explicada nos meses de janeiro e fevereiro de 1992, pela implantação apenas parcial do sistema no conjunto das maternidades, e no total de nascimentos de 1994, especialmente para o 
Figura 1

Distribuição dos nascidos vivos residentes em Belo Horizonte, por mês, 1992-1994.

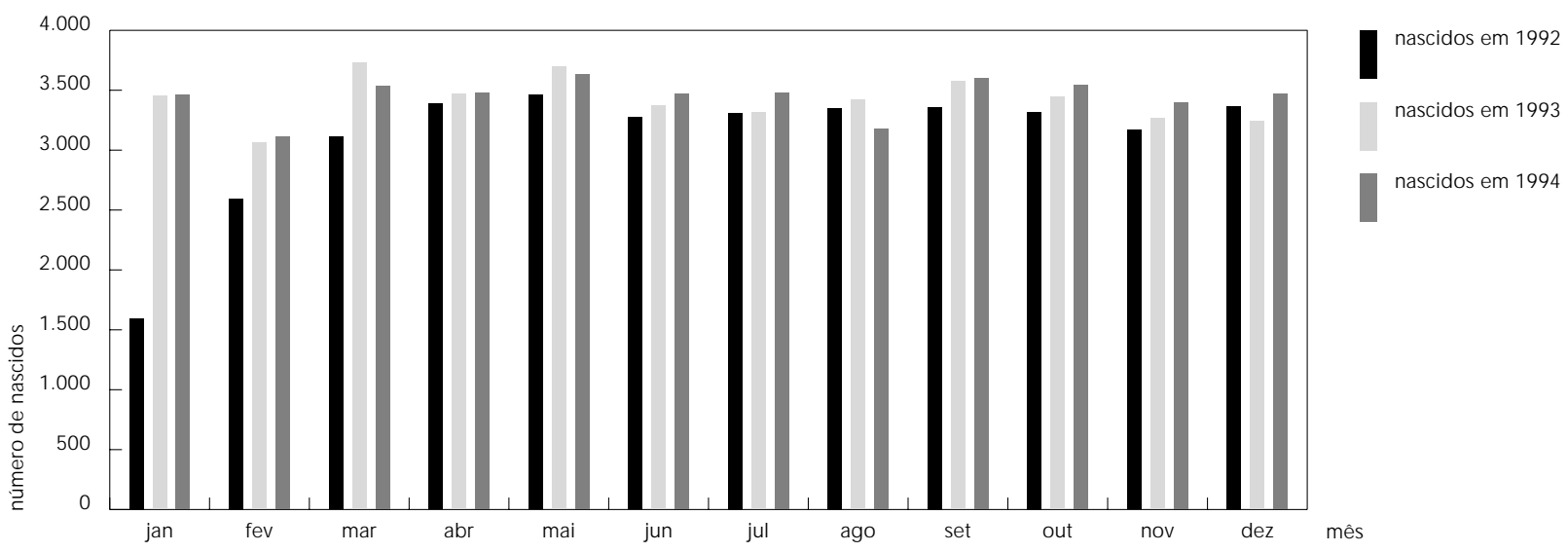

Fonte: Núcleo de Epidemiologia/SMSA - BH, Sinasc - MS.

DS Barreiro, pela inclusão de nascidos vivos em outros municípios da região metropolitana e residentes em Belo Horizonte.

A partir de 1993, através da melhoria das delimitações das áreas de abrangência das unidades básicas de saúde (Centros de Saúde) nos Distritos Sanitários e da utilização do georreferenciamento, pôde-se notar uma diminuição dos nascidos vivos com endereço ignorado no Município.

A análise dos nascidos vivos por local de ocorrência mostra que este é predominantemente hospitalar, variando de $99,4 \%$ a $99,8 \%$ dos nascimentos (Tabela 2).

Em relação ao sexo dos recém-nascidos observa-se maior número destes sendo do sexo masculino, com razão de masculinidade de 1,04 (Tabela 2).

O percentual de crianças com baixo peso ao nascer $(<2.500 \mathrm{~g}$ ) variou de 9,9 a 10,7 nos três anos (Tabela 2), tendo sido semelhante ao encontrado em outros estudos latino-americanos (Schwarcz et al., 1984) e na cidade de Pelotas (Victora et al., 1988).

Observa-se maior concentração dos nascidos vivos na faixa entre 37-41 semanas de gestação, variando o percentual de prematuros entre 5,2 e 5,8 (Tabela 2).

Faz-se necessário refletir, frente ao número de nascidos vivos prematuros encontrados, sobre a qualidade da assistência pré-natal prestada, visto que, dentre os vários fatores que levam à prematuridade, al guns poderiam ser evitados com acompanhamento pré-natal satisfatório.
Tabela 1

Distribuição dos nascidos vivos residentes em Belo Horizonte, por Distrito Sanitário, 1992-1994.

\begin{tabular}{lrrrrrr}
\hline \multirow{2}{*}{ Distrito } & \multicolumn{2}{c}{1992} & \multicolumn{2}{c}{1993} & \multicolumn{2}{c}{1994} \\
& Freq. & $\%$ & Freq. & $\%$ & Freq. & $\%$ \\
\hline Barreiro & 3.500 & 9,5 & 3.745 & 9,1 & 4.550 & 11,0 \\
Centro-Sul & 3.501 & 9,5 & 3.922 & 9,6 & 3.805 & 9,2 \\
Leste & 4.509 & 12,2 & 5.409 & 13,2 & 5.257 & 12,7 \\
Nordeste & 4.601 & 12,5 & 5.310 & 12,9 & 5.261 & 12,7 \\
Noroeste & 6.041 & 16,4 & 6.479 & 15,8 & 6.323 & 15,3 \\
Norte & 3.885 & 10,6 & 3.460 & 8,4 & 3.769 & 9,1 \\
Oeste & 4.363 & 11,8 & 4.942 & 12,1 & 5.124 & 12,4 \\
Pampulha & 2.211 & 6,0 & 2.450 & 6,0 & 2.218 & 5,4 \\
Venda Nova & 4.257 & 11,5 & 5.287 & 12,9 & 4.966 & 12,0 \\
Ignorados & 404 & - & 166 & - & 17 & - \\
Total & 37.272 & 100,0 & 41.170 & 100,0 & 41.290 & 100,0 \\
\hline
\end{tabular}

Fonte: Núcleo de Epidemiologia/SMSA-BH, Sinasc - MS.

O bservação: Percentuais excluindo-se os ignorados.

Nota-se pela Tabela 2 que o percentual de gestações múltiplas ficou entre 1,9e 2,1.

Em relação à idade materna, observa-se que houve predomínio na faixa etária de 20-34 anos, com percentual de mães adolescentes (< 20 anos ) variando de 13,5 a 14,7 (Tabela 2). Este percentual foi semelhante ao da cidade de Pelotas em 1982 e bem superior ao encontrado na Inglaterra e País de Gales (Victora et al., 1988).

Apesar do predomínio do parto espontâneo, constatou-se um alto percentual de parto operatório, sendo esta variação de 40,5 a 42,7 
Tabela 2

Caracterização dos nascidos vivos residentes em Belo Horizonte, 1992-1994.

\begin{tabular}{|c|c|c|c|c|c|c|}
\hline & \multicolumn{2}{|c|}{1992} & \multicolumn{2}{|c|}{1993} & \multicolumn{2}{|c|}{1994} \\
\hline & Freq. & $\%$ & Freq. & $\%$ & Freq. & $\%$ \\
\hline \multicolumn{7}{|c|}{ Local de ocorrência } \\
\hline Hospital & 36.524 & 99,6 & 40.763 & 99,4 & 41.134 & 99,8 \\
\hline Domicílio & 152 & 0,4 & 255 & 0,6 & 96 & 0,2 \\
\hline \multicolumn{7}{|l|}{ Sexo } \\
\hline Masculino & 18.924 & 51,0 & 20.929 & 51,0 & 20.967 & 50,9 \\
\hline Feminino & 18.209 & 49,0 & 20.079 & 49,0 & 20.215 & 49,1 \\
\hline \multicolumn{7}{|l|}{ Peso ao nascer } \\
\hline$<2.500 \mathrm{~g}$ & 3.653 & 9,9 & 4.390 & 10,7 & 4.372 & 10,6 \\
\hline$\geq 2.500 \mathrm{~g}$ & 33.358 & 90,1 & 36.465 & 89,3 & 36.787 & 89,4 \\
\hline \multicolumn{7}{|c|}{ Duração gestação } \\
\hline$<37$ semanas & 1.908 & 5,2 & 2.262 & 5,6 & 2.353 & 5,8 \\
\hline$\geq 37$ semanas & 34.739 & 94,8 & 38.219 & 94,4 & 38.470 & 94,2 \\
\hline \multicolumn{7}{|l|}{ Tipo gravidez } \\
\hline Única & 36.226 & 97,9 & 40.121 & 98,1 & 40.258 & 97,9 \\
\hline Múltipla & 762 & 2,1 & 798 & 1,9 & 878 & 2,1 \\
\hline \multicolumn{7}{|l|}{ Idade materna } \\
\hline$<20$ anos & 4.851 & 13,5 & 5.705 & 14,1 & 6.024 & 14,7 \\
\hline $20-34$ anos & 27.767 & 77,0 & 31.020 & 76,4 & 31.046 & 75,7 \\
\hline$>35$ anos & 3.439 & 9,5 & 3.869 & 9,5 & 3.928 & 9,6 \\
\hline \multicolumn{7}{|l|}{ Tipo parto } \\
\hline Espontâneo & 20.875 & 56,4 & 23.019 & 56,3 & 22.616 & 54,9 \\
\hline Operatório & 15.015 & 40,5 & 16.748 & 40,9 & 17.560 & 42,7 \\
\hline Fórceps & 156 & 3,1 & 1.152 & 2,8 & 966 & 2,4 \\
\hline \multicolumn{7}{|c|}{ Grau instrução materno } \\
\hline$<8$ anos & 20.758 & 58,2 & 23.473 & 59,2 & 22.885 & 58,6 \\
\hline$\geq 8$ anos & 14.909 & 41,8 & 16.180 & 40,8 & 16.167 & 41,4 \\
\hline
\end{tabular}

Fonte : Núcleo de Epidemiologia/SMSA-BH, Sinasc - MS

Observações:

1 - Total de nascidos vivos: $1992=37.272 ; 1993=41.170 ; 1994=41.290$.

2 - Percentuais excluindo-se os ignorados.

(Tabela 2). Como descrito na literatura, os índices de cesariana no Brasil e em alguns outros países vêm aumentando nas últimas décadas, não podendo ser explicados apenas como esforço para a melhoria dos resultados perinatais (Faundes \& Cecatti, 1991; Victora et al., 1988).

O grau de instrução materno pode ser visto como um indicador de condição social, sendo que o maior grau de instrução facilita acesso a emprego e melhoria da posição sócio-econômica da família (Ayçaguer \& Macho, 1990; Macharelli \& Oliveira, 1991; Victora et al., 1988). No presente estudo, $58,2 \%$ a $59,2 \%$ das mulheres assistidas não possuíam 10 grau completo (Tabela 2).

\section{Discussão}

Após três anos de implantação do Sinasc, verifica-se que o perfil de nascidos vivos não difere significativamente entre os anos, nem em relação a outros municípios do mesmo porte de $\mathrm{BH}$.

O grande avanço e contribuição que este sistema trouxe, além do denominador mais fidedigno para o cálculo da taxa de mortalidade infantil, reside no fato de deixar disponíveis informações rotineiras, ágeis, permanentes e descentralizadas para os Centros de Saúde, Distritos Sanitários, hospitais e serviços públicos de educação, saúde, planejamento etc, as- 
sim como para os serviços privados, possibilitando a retirada de diferentes indicadores, necessários ao planejamento e avaliação de ações no Município, bem como o seu uso pelas instituições docentes.

Um exemplo disso é a disponibilidade de informação sobre a utilização de serviços nos diversos municípios que compõem uma área conurbada como a região metropolitana de $\mathrm{BH}$, permitindo melhor alocação de recursos humanos e materiais. Outro exemplo é a possibilidade de desagregação das informações por pequenas áreas que sejam de responsabilidade sanitária de determinada unidade e equipe de saúde, sugerindo a possibilidade de sua utilização para intervenções específicas e diferenciadas em determinados grupos populacionais e microáreas de risco, como já foi iniciado em Belo Horizonte na abordagem da mortalidade infantil.

\section{Agradecimentos}

Agradecemos a Dra. Suzana F. Gontijo Assunção pela tradução.

\section{Conclusões}

O uso do Sinasc e a análise do perfil dos nascidos vivos no Município de Belo Horizonte permitem identificar que:

- A quase totalidade dos partos ocorre em hospitais.

- O percentual de baixo peso ao nascer e de prematuros corroboram outros estudos já realizados no Brasil.

- Há um alto percentual de partos operatórios, indicando a necessidade de outros estudos para aprofundar a análise desta situação, principal mente no que diz respeito à morbimortalidade materna e infantil e ao custo das internações.

- Há um grande número de gravidez em adolescentes, mostrando a necessidade urgente de um trabalho mais eficaz com este segmento da população em todos os seus aspectos.

- É possível haver desagregação das informações por pequenas áreas que sejam de responsabilidade sanitária de determinada unidade e equipe de saúde.

- O Sinasc é um instrumento poderoso de informação na área da sáude, devendo ser aprimorado e seu uso difundido para outros Municípios.

\section{Referências}

AYÇAGUER, L. C. S. \& MACHO, E. D., 1990. Mortalidad infantil y condiciones higiénico-sociales en las Américas. Un estudio de correlaciones. Revista de Saúde Pública, 24:473-480.

FAUNDES, A. \& CECATTI, J. G., 1991. Operação cesárea no Brasil. Incidência, tendências, causas, conseqüências e propostas de ação. Cadernos de SaúdePública, 7:150-173.

MACHARELLI, C. A. \& OLIVEIRA, L. R., 1991. Perfil do risco de óbito de crianças menores de um ano residentes em localidade do Estado de São Paulo, Brasil, 1987. Revista de Saúde Pública, 25:121128.

SCHWARCZ, R.; DIAZ, A. G.; FESCINA, R.; ROSSELLO, J. L. D. \& MARTEL, M., 1984. Bajo peso al nacer y mortalidad perinatal en maternidades da América Latina. In: Salud Materno Infantil y Atención Primaria en las Américas (Organización Panamericana de la Salud, org.), pp. 105-117. México DF: OPAS, Publicación Científica 461,

VICTORA, C. G.; BARROS, F. C. \& VAUGHAN, J. P., 1988. Epidemiologia da Desigual dade. São Paulo: Hucitec. 